\title{
An asymptotic membrane-like theory for long wave motion in a pre-stressed elastic plate
}

\author{
By Aleksey V. Pichugin and Graham A. Rogerson \\ Department of Computer and Mathematical Sciences, \\ University of Salford, Salford M5 4 WT. UK.
}

\begin{abstract}
An asymptotically consistent two-dimensional theory is developed to help elucidate dynamic response in finitely deformed layers. The layers are composed of incompressible elastic material, with the theory appropriate for long wave motion associated with the fundamental mode and derived in respect of the most general appropriate strain energy function. Leading order and refined higher order equations for the mid-surface deflection are derived. In the case of zero normal initial static stress and in-plane tension, the leading order equation reduces to the classical membrane equation, with its refined counterpart also being obtained. The theory is applied to a one-dimensional edge loading problem for a semi-infinite plate. In doing so the leading order and higher order governing equations are used as inner and outer asymptotic expansions, the latter valid within the vicinity of the associated quasi-front. A solution is derived by using the method of matched asymptotic expansions.
\end{abstract}

Keywords: pre-stress, elastic plates, waves, asymptotics, membrane.

\section{Introduction}

The constitutive complications afforded by the existence of a finite primary deformation, prior to any dynamic loading, make the derivation of lower-dimensional (asymptotically consistent) models for layered pre-stressed structures highly attractive. In the static case, engineers have used lower-dimensional structural theories for many years now, the most widely known being Kirchhoff plate theory, Kirchhoff-Love shell theory and the refined Timoshenko-Reissner theories. In the case of static problems, only one type of asymptotic 
approximation, coupled with careful boundary layer analysis near the edge, is required, see for example Green (1962). In the dynamic case, high frequency motion will be an additional feature of the problem. Moreover, high frequency motion will in general consist of both long and short wave contributions. A full detailed account of the asymptotic methodology required to determine the dynamic response of thin-walled elastic structures may be found in Kaplunov et al. (1998). In our present paper we present a two-dimensional asymptotic dynamic model to describe three-dimensional long wave low frequency motion in a pre-stressed, incompressible elastic plate. This model is a generalization of a previously developed one-dimensional model, see Kaplunov et al. (2000).

Over the last few years there have been a number of studies aimed at elucidating aspects of the dynamic response of finitely deformed, incompressible elastic layers and half-spaces, see for example Ogden \& Sotiropoulos (1995); Dowaikh \& Ogden (1990); Rogerson \& Sandiford (1997). These studies have in part been motivated by the increasing use of rubber-like materials with an ability to sustain large deformation prior to failure. In the case of long wave motion, one-dimensional asymptotic theories for the plane strain problem have been constructed, see Kaplunov et al. (2001) and Kaplunov et al. (2000). In the three-dimensional case, asymptotic models have been constructed to describe symmetric and anti-symmetric long wave high frequency motion, see Pichugin \& Rogerson (2001a) and Pichugin \& Rogerson (2001b). However, no asymptotically consistent lower dimensional model has previously been presented to analyse three-dimensional long wave low frequency motion in an elastic layer. Long wave low frequency motion is associated with the fundamental mode at low wave number and is the most important type of motion in structural analysis. Accordingly, in this paper we will derive an asymptotic model for long wave low frequency anti-symmetric motion in a pre-stressed incompressible elastic layer. At leading order, a second order hyperbolic governing equation for the mid-surface deflection is obtained. This equation has no counterpart in the corresponding classical theory and its occurrence indicates that the pre-stress makes the plate stiffer and precludes bending. Associated with this leading order governing equation is a discontinuous wave front, usually termed a quasi-front. In order to smooth this discontinuity, and clarify whether the front is advancing or receding, a refined higher order theory is established.

In section 2 of this paper the governing equations associated with small time-dependent motion superimposed upon a homogeneous primary deformation are briefly reviewed, specif- 
ically in respect of a general incompressible (initially isotropic) elastic solid. The dispersion relation is derived in section 3 and low wave number approximations for the fundamental mode, giving phase speed as a function of wave number, are established. These approximations are used to obtain the relative orders of displacement components and indicate that for anti-symmetric long wave low frequency motion the normal displacement is asymptotically leading. Motivated by the relative orders of displacement components, and with appropriate scales introduced for time and space variables, a hierarchy of approximate equations and appropriate boundary conditions is set up in section 4 . The leading order problem is solved to enable all leading order displacement components to be expressed in terms of the leading order mid-surface deflection. An equation for the leading order mid-surface deflection is obtained from the second order problem, as are second order solutions for other displacement components in terms of both the leading order mid-surface deflection and its second order correction. A refined higher order equation for the mid-surface deflection is obtained from the third order problem.

An interesting feature of the leading order equation for the mid-surface deflection is that it may be used to obtain the classical equation for wave propagation in a membrane. Specifically, requiring that the normal principal Cauchy pre-stress vanishes and that the corresponding in-plane principal stresses are pure tensions does this. It would seem that this is the first time that this equation has been derived by asymptotic analysis of the full three-dimensional equations. A refined higher order membrane equation is also obtained. As one might expect, the coefficients of this equation are not expressible in terms of the two in-plane tensions and material density. For the refined equation the coefficients are functions of material parameters and membrane thickness, in addition to in-plane tensions and membrane density. In the case of both the leading order and the higher order refined equations for the mid-surface deflection, the associated dispersion relations agree exactly with corresponding expansions of the dispersion relation associated with the full threedimensional problem.

Finally, in section (5), the theory is applied to considering the model problem of shock edge loading of a semi-infinite plate. Specifically, a one-dimensional impact problem is formulated and the solution sought for a variety of angles of propagation. The leading order equation for the mid-surface deflection is employed as an outer asymptotic expansion, with the refined higher order equation used as a inner expansion valid in the vicinity of the quasi- 
front. The boundary condition on the edge is imposed on the outer solution and the method of matched asymptotic expansions used to compensate for the required boundary condition for the inner expansion. For different angles of propagation, the solutions show that both receding and advancing fronts are possible. This phenomenon is very clearly illustrated numerically by some three-dimensional projections showing the transition through different angles of propagation.

\section{Governing equations}

Our concern in this paper is a finitely deformed layer, composed of incompressible elastic material. We place the origin $O$ of a Cartesian coordinate system $O x_{1} x_{2} x_{3}$ in the mid-plane of the layer with axis $O x_{2}$ orthogonal to the layer and focus attention on finite primary deformations of the form

$$
x_{1}=\lambda_{1} X_{1}, \quad x_{2}=\lambda_{2} X_{2}, \quad x_{3}=\lambda_{3} X_{3},
$$

in which $\lambda_{m}(m \in\{1,2,3\})$ are the principal stretches of the primary deformation and $X_{m}$ and $x_{m}(m \in\{1,2,3\})$ coordinates of a typical particle in the initial and the deformed states, respectively. We will concentrate our study on small time-dependent incremental motion superimposed on the finitely deformed layer. The equations of incremental motion for a pre-stressed incompressible elastic solid may be linearized, which has previously been done, see e.g. Dowaikh \& Ogden (1990), to obtain

$$
B_{m i l k} u_{k, l m}-p_{t, i}=\rho \ddot{u}_{i}
$$

where $B_{\text {milk }}$ are components of the elasticity tensor, $\mathbf{u}$ is the superimposed infinitesimal displacement, $\rho$ the material density and a comma and dot denote differentiation with respect to $x_{i}$ and time $t$, respectively. The scalar quantity $p_{t}=p_{t}(\mathbf{x}, t)$ is a time-dependent component of workless reaction stress $p \equiv \bar{p}+p_{t}$, with $\bar{p}$ being a reaction stress in the finitely deformed state. This reaction stress, usually referred to as pressure, is generated in order to maintain the linearized incompressibility constraint

$$
u_{i, i}=0
$$

Note, that the use of summation convention has been made in (2.2) and (2.3) (we will always explicitly state occasions where this convention has been applied). A linearized measure of 
surface traction increment has also been previously obtained in the component form

$$
\tau_{i}=B_{m i l k} u_{k, l} n_{m}+\bar{p} u_{m, i} n_{m}-p_{t} n_{i}
$$

with $\boldsymbol{n}$ the unit outward normal to a material surface in the deformed layer and the summation convention has been utilized, see e.g. Dowaikh \& Ogden (1990). Further description of the derivation of the equations (2.2)-(2.4) is given in Rogerson \& Fu (1995).

\section{(a) Secular equation}

It has been shown in Ogden (1984), that for an incompressible (initially) isotropic elastic body all non-zero components of the elasticity tensor $\boldsymbol{B}$, referred to the previously defined coordinate system, have one of the forms $B_{i i j j}, B_{i j i j}$ or $B_{i j j i}$, where $B_{i i j j}=B_{j j i i}$ and $B_{i j j i}=B_{j i i j},(i, j \in\{1,2,3\})$. Therefore, the linearized equations of motion (2.2) may be written explicitly in the form

$$
\begin{aligned}
B_{1111} u_{1,11}+\left(B_{1122}+B_{2112}\right) u_{2,12} & +\left(B_{1133}+B_{3113}\right) u_{3,13} \\
& +B_{2121} u_{1,22}+B_{3131} u_{1,33}-p_{t, 1}=\rho \ddot{u}_{1}, \\
\left(B_{2211}+B_{1221}\right) u_{1,12}+B_{2222} u_{2,22} & +\left(B_{2233}+B_{3223}\right) u_{3,23} \\
& +B_{1212} u_{2,11}+B_{3232} u_{2,33}-p_{t, 2}=\rho \ddot{u}_{2}, \\
\left(B_{3311}+B_{1331}\right) u_{1,13}+\left(B_{3322}+\right. & \left.B_{2332}\right) u_{2,23}+B_{3333} u_{3,33} \\
& +B_{1313} u_{3,11}+B_{2323} u_{3,22}-p_{t, 3}=\rho \ddot{u}_{3} .
\end{aligned}
$$

The solution of the equations of motion (2.5)-(2.7) will now be sought in form of a traveling harmonic wave

$$
\left(u_{1}, u_{2}, u_{3}, p_{t}\right)=\left(U_{1}, U_{2}, U_{3}, k P\right) e^{k q x_{2}} e^{i k\left(x_{1} c_{\theta}+x_{3} s_{\theta}-v t\right)},
$$

where $k$ is the wave number, $v$ the wave speed, $\left(c_{\theta}, 0, s_{\theta}\right) \equiv(\cos \theta, 0, \sin \theta)$ is the in-plane projection of the wave normal and the parameter $q$ is to be determined from the governing equations. Since the analogous plain-strain problem has previously been analysed by Kaplunov et al. (2000), we seek to generalize the problem to the three-dimensional case and tacitly assume that $c_{\theta} \neq 0$ and $s_{\theta} \neq 0$. Substituting the solutions (2.8) into the equations of motion (2.5)-(2.7) and the incompressibility condition (2.3) we obtain a system of 4 linear homogeneous equations. This system possesses a non-trivial solution provided the 
determinant of its coefficients is equal to zero, which yields a necessary condition, usually referred to as the secular equation

$$
\gamma_{21} \gamma_{23} q^{6}+\left(\left(\gamma_{21}+\gamma_{23}\right) \bar{v}^{2}-c_{1}\right) q^{4}+\left(\bar{v}^{4}-c_{2} \bar{v}^{2}+c_{3}\right) q^{2}-\left(\bar{v}^{2}-c_{4}\right)\left(\bar{v}^{2}-c_{5}\right)=0
$$

in which $\bar{v} \equiv \sqrt{\rho} v$ is the scaled wave speed and

$$
\begin{aligned}
& c_{1}=\left(2 \beta_{23} \gamma_{21}+\gamma_{23} \gamma_{31}\right) s_{\theta}^{2}+\left(2 \beta_{12} \gamma_{23}+\gamma_{21} \gamma_{13}\right) c_{\theta}^{2}, \\
& c_{2}=\left(2 \beta_{23}+\gamma_{21}+\gamma_{31}\right) s_{\theta}^{2}+\left(2 \beta_{12}+\gamma_{23}+\gamma_{13}\right) c_{\theta}^{2}, \\
& c_{3}=\left(4 \beta_{12} \beta_{23}+\gamma_{21} \gamma_{12}+\gamma_{23} \gamma_{32}+\gamma_{13} \gamma_{31}-\mu_{13}^{2}\right) s_{\theta}^{2} c_{\theta}^{2} \\
& \quad+\left(2 \beta_{23} \gamma_{31}+\gamma_{21} \gamma_{32}\right) s_{\theta}^{4}+\left(2 \beta_{12} \gamma_{13}+\gamma_{23} \gamma_{12}\right) c_{\theta}^{4}, \\
& c_{4}=\gamma_{32} s_{\theta}^{2}+\gamma_{12} c_{\theta}^{2}, \quad c_{5}=\gamma_{31} s_{\theta}^{4}+2 \beta_{13} s_{\theta}^{2} c_{\theta}^{2}+\gamma_{13} c_{\theta}^{4} .
\end{aligned}
$$

The material parameters $\gamma_{i j}, \beta_{i j}$ and $\mu_{i j}, i, j \in\{1,2,3\}$ are defined through the components of the elasticity tensor $\boldsymbol{B}$ as follows

$$
\begin{gathered}
\gamma_{i j}=B_{i j i j}, \quad b_{i j}=B_{i i i i}-B_{i i j j}-B_{i j j i}, \quad 2 \beta_{i j}=b_{i j}+b_{j i}, \quad i \neq j, \\
\mu_{i j}=\beta_{i j}-\beta_{i k}-\beta_{j k}, \quad i<j, \quad k \notin\{i, j\}, \quad i, j, k \in\{1,2,3\} .
\end{gathered}
$$

The three roots of the cubic (in $q^{2}$ ) secular equation (2.9), denoted by $q_{1}^{2}, q_{2}^{2}$ and $q_{3}^{2}$, will always satisfy the following equalities

$$
\begin{gathered}
q_{1}^{2}+q_{2}^{2}+q_{3}^{2} \equiv-\frac{\left(\gamma_{21}+\gamma_{23}\right) \bar{v}^{2}-c_{1}}{\gamma_{21} \gamma_{23}}, \quad q_{1}^{2} q_{2}^{2} q_{3}^{2} \equiv \frac{\left(\bar{v}^{2}-c_{4}\right)\left(\bar{v}^{2}-c_{5}\right)}{\gamma_{21} \gamma_{23}}, \\
q_{1}^{2} q_{2}^{2}+q_{1}^{2} q_{3}^{2}+q_{2}^{2} q_{3}^{2} \equiv \frac{\bar{v}^{4}-c_{2} \bar{v}^{2}+c_{3}}{\gamma_{21} \gamma_{23}}
\end{gathered}
$$

When the roots $q_{1}^{2}, q_{2}^{2}$ and $q_{3}^{2}$ are all distinct and not equal to zero, any solution for $u_{1}$, $u_{2}, u_{3}$ or $p_{t}$ can be represented as a superposition of the six linearly independent functions $\exp \left(k q_{m} x_{2}\right)$ and $\exp \left(-k q_{m} x_{2}\right), m \in\{1,2,3\}$ (in order to avoid possible ambiguity, we hereafter assume that the real part of $q_{m}$ is positive). In this paper we specifically consider motion for which $u_{2}$ is an even function of the normal coordinate $x_{2}$. This type of motion is usually referred to as flexural or anti-symmetric and solutions for $u_{1}, u_{2}, u_{3}$ or $p_{t}$ are then representable as superpositions of three linearly independent functions. The coefficients of these superpositions may all be expressed in terms of three disposable constants $U_{2}^{(m)}$, 
$m \in\{1,2,3\}$, as follows

$$
\begin{array}{ll}
u_{1}=\sum_{m=1}^{3} \frac{i q_{m} \mathcal{U}_{1}\left(q_{m}, \bar{v}\right) c_{\theta}}{\mathcal{V}\left(q_{m}, \bar{v}\right)} S_{m}\left(x_{2}\right) U_{2}^{(m)}, & u_{2}=\sum_{m=1}^{3} C_{m}\left(x_{2}\right) U_{2}^{(m)}, \\
u_{3}=\sum_{m=1}^{3} \frac{i q_{m} \mathcal{U}_{3}\left(q_{m}, \bar{v}\right) s_{\theta}}{\mathcal{V}\left(q_{m}, \bar{v}\right)} S_{m}\left(x_{2}\right) U_{2}^{(m)}, & p_{t}=\sum_{m=1}^{3} \frac{q_{m} \mathcal{P}\left(q_{m}, \bar{v}\right)}{\mathcal{V}\left(q_{m}, \bar{v}\right)} S_{m}\left(x_{2}\right) U_{2}^{(m)},
\end{array}
$$

in which $S_{m}\left(x_{2}\right) \equiv \sinh \left(k q_{m} x_{2}\right), C_{m}\left(x_{2}\right) \equiv \cosh \left(k q_{m} x_{2}\right)$ and

$$
\begin{aligned}
\mathcal{U}_{1}\left(q_{m}, \bar{v}\right) & =\gamma_{23} q_{m}^{2}+\mu_{12} s_{\theta}^{2}-\gamma_{13} c_{\theta}^{2}+\bar{v}^{2}, \quad \mathcal{U}_{3}\left(q_{m}, \bar{v}\right)=\gamma_{21} q_{m}^{2}-\gamma_{31} s_{\theta}^{2}+\mu_{23} c_{\theta}^{2}+\bar{v}^{2} \\
\mathcal{P}\left(q_{m}, \bar{v}\right) & =\mathcal{U}_{1}\left(q_{m}, \bar{v}\right) \mathcal{U}_{3}\left(q_{m}, \bar{v}\right)+\left(b_{31}-b_{32}\right) \mathcal{U}_{1}\left(q_{m}, \bar{v}\right) c_{\theta}^{2}+\left(b_{13}-b_{12}\right) \mathcal{U}_{3}\left(q_{m}, \bar{v}\right) s_{\theta}^{2} \\
\mathcal{V}\left(q_{m}, \bar{v}\right) & =\left(\gamma_{21} s_{\theta}^{2}+\gamma_{23} c_{\theta}^{2}\right) q_{m}^{2}+\bar{v}^{2}-c_{5} .
\end{aligned}
$$

The above representation of $\mathcal{P}\left(q_{m}, \bar{v}\right)$ can only be obtained with help of the equality

$$
q_{m}^{2} \mathcal{U}_{1}\left(q_{m}, \bar{v}\right) \mathcal{U}_{3}\left(q_{m}, \bar{v}\right)=-\left(\mu_{13} q^{2}+\gamma_{32} s_{\theta}^{2}+\gamma_{12} c_{\theta}^{2}-\bar{v}^{2}\right) \mathcal{V}\left(q_{m}, \bar{v}\right)
$$

which is a direct consequence of the above definitions of functions $\mathcal{U}_{1}\left(q_{m}, \bar{v}\right), \mathcal{U}_{3}\left(q_{m}, \bar{v}\right)$ and the secular equation (2.9).

\section{Dispersion relation}

The upper and lower surfaces of the layer are defined in the specified system of coordinates by the outward normals $(0,1,0)$ and $(0,-1,0)$, respectively. Therefore, the measure $(2.4)$ of surface traction on a layer surface may be given in the component form

$$
\begin{aligned}
& \tau_{1}=B_{2121} u_{1,2}+\left(B_{2112}+\bar{p}\right) u_{2,1}, \\
& \tau_{2}=B_{2211} u_{1,1}+\left(B_{2222}+\bar{p}\right) u_{2,2}+B_{2233} u_{3,3}-p_{t}, \\
& \tau_{3}=\left(B_{2332}+\bar{p}\right) u_{2,3}+B_{2323} u_{3,2} .
\end{aligned}
$$

The static pressure component $\bar{p}$ will be eliminated in the subsequent analysis in favour of the normal Cauchy stress component $\sigma_{2}$. For a primary deformation given by $(2.1)$ the coordinate axes are coincident with the principal axes of pre-stress and, consequently, $\bar{p}$ and $\sigma_{2}$ are related through $\bar{p}=\gamma_{21}-B_{1221}-\sigma_{2}=\gamma_{23}-B_{2332}-\sigma_{2}$, which is a consequence, see Ogden (1984) and Spencer (1980), of the more general result

$$
\sigma_{i}=B_{i j i j}-B_{i j j i}-\bar{p}, \quad i \neq j, \quad i, j \in\{1,2,3\}
$$


A dispersion relation associated with the propagation of infinitesimal harmonic waves in a pre-stressed incompressible elastic layer will now be derived. Inserting the displacement and pressure representations (2.12) into (3.1)-(3.3) and imposing zero surface traction boundary conditions, a system of six homogeneous linear equations is obtained. Three of the equations are satisfied identically for flexural motion, while the remaining three may be written as

$$
\begin{gathered}
\sum_{m=1}^{3} \frac{\mathcal{T}_{1}\left(q_{m}, \bar{v}\right)}{\mathcal{V}\left(q_{m}, \bar{v}\right)} C_{m}(h) U_{2}^{(m)}=0 \\
\sum_{m=1}^{3} \frac{q_{m} \mathcal{T}_{2}\left(q_{m}, \bar{v}\right)}{\mathcal{V}\left(q_{m}, \bar{v}\right)} S_{m}(h) U_{2}^{(m)}=0 \\
\sum_{m=1}^{3} \frac{\mathcal{T}_{3}\left(q_{m}, \bar{v}\right)}{\mathcal{V}\left(q_{m}, \bar{v}\right)} C_{m}(h) U_{2}^{(m)}=0
\end{gathered}
$$

where $h$ is the half-thickness of the layer and

$$
\begin{aligned}
& \mathcal{T}_{1}\left(q_{m}, \bar{v}\right)=\gamma_{21} \mathcal{U}_{1}\left(q_{m}, \bar{v}\right) q_{m}^{2}+g_{1} \mathcal{V}\left(q_{m}, \bar{v}\right), \\
& \mathcal{T}_{2}\left(q_{m}, \bar{v}\right)=\left(g_{1}-\mu_{13}\right) \mathcal{U}_{1}\left(q_{m}, \bar{v}\right) c_{\theta}^{2}+\left(g_{3}-\mu_{13}\right) \mathcal{U}_{3}\left(q_{m}, \bar{v}\right) s_{\theta}^{2}-\mathcal{U}_{1}\left(q_{m}, \bar{v}\right) \mathcal{U}_{3}\left(q_{m}, \bar{v}\right), \\
& \mathcal{T}_{3}\left(q_{m}, \bar{v}\right)=\gamma_{23} \mathcal{U}_{3}\left(q_{m}, \bar{v}\right) q_{m}^{2}+g_{3} \mathcal{V}\left(q_{m}, \bar{v}\right),
\end{aligned}
$$

in which

$$
g_{i} \equiv \gamma_{2 i}-\sigma_{2}, \quad G_{i} \equiv 2 \gamma_{2 i}-\sigma_{2}, \quad i \in\{1,3\}
$$

The homogeneous system of three linear equations (3.5) possesses a non-trivial solution provided the determinant of its coefficients vanishes, yielding a dispersion relation for flexural waves, which after omitting several non-dispersive factors takes the form

$$
\begin{aligned}
\left(q_{2}^{2}-q_{3}^{2}\right) \mathcal{T}_{2}\left(q_{1}, \bar{v}\right) \mathcal{H}\left(q_{2}, q_{3}, \bar{v}\right) q_{1} T_{1}(h)- & \left(q_{1}^{2}-q_{3}^{2}\right) \mathcal{T}_{2}\left(q_{2}, \bar{v}\right) \mathcal{H}\left(q_{1}, q_{3}, \bar{v}\right) q_{2} T_{2}(h) \\
& +\left(q_{1}^{2}-q_{2}^{2}\right) \mathcal{T}_{2}\left(q_{3}, \bar{v}\right) \mathcal{H}\left(q_{1}, q_{2}, \bar{v}\right) q_{3} T_{3}(h)=0
\end{aligned}
$$

with $T_{m}(h) \equiv \tanh \left(k q_{m} h\right), m \in\{1,2,3\}$

$$
\begin{aligned}
\mathcal{H}\left(q_{i}, q_{j}, \bar{v}\right) & =\gamma_{21} \gamma_{23} \mathcal{H}_{1}(\bar{v}) q_{i}^{2} q_{j}^{2}+\left(\bar{v}^{2}-c_{5}\right)\left(\gamma_{21} \gamma_{23}\left(\gamma_{23}-\gamma_{21}\right)\left(q_{i}^{2}+q_{j}^{2}\right)-\mathcal{H}_{2}(\bar{v})\right) \\
\mathcal{H}_{1}(\bar{v}) & =\left(\gamma_{23}-\gamma_{21}\right)\left(\gamma_{23} c_{\theta}^{2}+\gamma_{21} s_{\theta}^{2}\right)+\gamma_{23} \mathcal{U}_{3}(0, \bar{v})-\gamma_{21} \mathcal{U}_{1}(0, \bar{v}) \\
\mathcal{H}_{2}(\bar{v}) & =g_{1} \gamma_{23} \mathcal{U}_{3}(0, \bar{v})-g_{3} \gamma_{21} \mathcal{U}_{1}(0, \bar{v})
\end{aligned}
$$

and the definition of $\mathcal{T}_{2}\left(q_{i}, \bar{v}\right)$ is given immediately after the system of equations $(3.5)$. The dispersion relation (3.6) was seemingly first derived, in slightly different notation, by Rogerson \& Sandiford (1999). 
(a) Long wave low frequency approximation

In order to discuss long wave motion it is convenient to introduce a small non-dimensional parameter $\eta$, defined as the ratio of a layer half-thickness $h$ to a typical wave length $l$, so $\eta \equiv h / l \equiv k h$. Previously published numerical solutions, see Rogerson \& Sandiford (1999), show that as the scaled wave number $k h \rightarrow 0$, there exists one finite phase speed limit, associated with the fundamental mode. Following the terminology adopted in Kaplunov et al. (1998), we will term this type of motion as long wave low frequency. Generally, for the fundamental mode we may assume that as $k h \rightarrow 0$, the roots of the secular equation (2.9) do not undergo significant changes and stay $O(1)$. These observations enable us to derive long wave low frequency approximations of the hyperbolic tangents $T_{m}(h)$ in the form of a power series

$$
T_{m}(h)=q_{m} \eta-\frac{1}{3} q_{m}^{3} \eta^{3}+O\left(\eta^{5}\right), \quad m \in\{1,2,3\} .
$$

On the substitution of the expansions (3.7) into the dispersion relation (3.6) we obtain an approximation with error $O\left(\eta^{4}\right)$, namely

$$
\begin{aligned}
& \left(q_{1}^{2}-q_{2}^{2}\right) \mathcal{T}_{2}\left(q_{3}, \bar{v}\right) \mathcal{H}\left(q_{1}, q_{2}, \bar{v}\right) q_{3}^{2}-\left(q_{1}^{2}-q_{3}^{2}\right) \mathcal{T}_{2}\left(q_{2}, \bar{v}\right) \mathcal{H}\left(q_{1}, q_{3}, \bar{v}\right) q_{2}^{2} \\
& \quad+\left(q_{2}^{2}-q_{3}^{2}\right) \mathcal{T}_{2}\left(q_{1}, \bar{v}\right) \mathcal{H}\left(q_{2}, q_{3}, \bar{v}\right) q_{1}^{2}-\frac{\eta^{2}}{3}\left\{\left(q_{1}^{2}-q_{2}^{2}\right) \mathcal{T}_{2}\left(q_{3}, \bar{v}\right) \mathcal{H}\left(q_{1}, q_{2}, \bar{v}\right) q_{3}^{4}\right. \\
& \left.\quad-\left(q_{1}^{2}-q_{3}^{2}\right) \mathcal{T}_{2}\left(q_{2}, \bar{v}\right) \mathcal{H}\left(q_{1}, q_{3}, \bar{v}\right) q_{2}^{4}+\left(q_{2}^{2}-q_{3}^{2}\right) \mathcal{T}_{2}\left(q_{1}, \bar{v}\right) \mathcal{H}\left(q_{2}, q_{3}, \bar{v}\right) q_{1}^{4}\right\}+O\left(\eta^{4}\right)=0
\end{aligned}
$$

Since we are interested in the behaviour in a vicinity of a finite wave speed limit, we seek a scaled wave speed approximation in the following form

$$
\bar{v}^{2}=\bar{v}_{0}^{2}+\bar{v}_{2}^{2} \eta^{2}+O\left(\eta^{4}\right)
$$

Inserting the expansion (3.9) into the approximation of the dispersion relation (3.8), we obtain an asymptotic equality which must be satisfied at each order. Note, that the parameters $q_{m}, m \in\{1,2,3\}$, which must satisfy the secular equation (2.9), may be eliminated through use of the relations $(2.10)-(2.11)$ taken in the form

$$
\begin{gathered}
q_{1}^{2}+q_{2}^{2}+q_{3}^{2}=-\frac{\left(\gamma_{21}+\gamma_{23}\right) \bar{v}_{0}^{2}-c_{1}}{\gamma_{21} \gamma_{23}}-\frac{\left(\gamma_{21}+\gamma_{23}\right) \bar{v}_{2}^{2}}{\gamma_{21} \gamma_{23}} \eta^{2}+O\left(\eta^{4}\right), \\
q_{1}^{2} q_{2}^{2}+q_{1}^{2} q_{3}^{2}+q_{2}^{2} q_{3}^{2}=\frac{\bar{v}_{0}^{4}-c_{2} \bar{v}_{0}^{2}+c_{3}}{\gamma_{21} \gamma_{23}}+\frac{\left(2 \bar{v}_{0}^{2}-c_{2}\right) \bar{v}_{2}^{2}}{\gamma_{21} \gamma_{23}} \eta^{2}+O\left(\eta^{4}\right), \\
q_{1}^{2} q_{2}^{2} q_{3}^{2}=\frac{\left(\bar{v}_{0}^{2}-c_{4}\right)\left(\bar{v}_{0}^{2}-c_{5}\right)}{\gamma_{21} \gamma_{23}}+\frac{\left(2 \bar{v}_{0}^{2}-c_{4}-c_{5}\right) \bar{v}_{2}^{2}}{\gamma_{21} \gamma_{23}} \eta^{2}+O\left(\eta^{4}\right) .
\end{gathered}
$$




\section{(b) Leading order}

The leading order term of the dispersion relation (3.8) may be factorized, yielding

$$
\begin{aligned}
& \gamma_{21} \gamma_{23}\left(q_{1}^{2}-q_{2}^{2}\right)\left(q_{1}^{2}-q_{3}^{2}\right)\left(q_{2}^{2}-q_{3}^{2}\right) \\
& \quad \times\left(\bar{v}_{0}^{2}-c_{5}\right)\left(\left(\gamma_{21}-\gamma_{23}\right) \bar{v}_{0}^{2}-f_{1}^{(0)}\right)\left(\bar{v}_{0}^{2}-\mathcal{F}_{c}^{(0)} c_{\theta}^{2}-\mathcal{F}_{s}^{(0)} s_{\theta}^{2}\right)=0,
\end{aligned}
$$

in which the equalities (3.10)-(3.12) were used and

$$
\begin{gathered}
f_{1}^{(0)}=\left(\mu_{23} \gamma_{23}+\gamma_{13} \gamma_{21}\right) c_{\theta}^{2}-\left(\mu_{12} \gamma_{21}+\gamma_{23} \gamma_{31}\right) s_{\theta}^{2}, \\
\mathcal{F}_{c}^{(0)}=\gamma_{12}-\frac{g_{1}^{2}}{\gamma_{21}}, \quad \mathcal{F}_{s}^{(0)}=\gamma_{32}-\frac{g_{3}^{2}}{\gamma_{23}} .
\end{gathered}
$$

Because all values $q_{m}, m \in\{1,2,3\}$, are distinct, the leading order term (3.13) can be equal to zero only when the leading order scaled wave speed $\bar{v}_{0}^{2}$ is equal to one of three possible limits. However, it may be shown numerically that only the limit $\bar{v}_{0}^{2}=\mathcal{F}_{c}^{(0)} c_{\theta}^{2}+\mathcal{F}_{s}^{(0)} s_{\theta}^{2}$ is dispersive.

In order to understand the nature of the other two (non-dispersive) phase speed limits, associated with (3.13), we resort back to the derivation of the displacements and pressure. Through use of the wave solution (2.8) and the incompressibility condition (2.3) the equations of motion (2.5) and (2.7) may be rearranged as

$$
\mathcal{V}(q, \bar{v}) U_{1}=i q c_{\theta} \mathcal{U}_{1}(q, \bar{v}) U_{2}, \quad \mathcal{V}(q, \bar{v}) U_{3}=i q s_{\theta} \mathcal{U}_{3}(q, \bar{v}) U_{2} .
$$

It follows from definitions given after $(2.12)$, that $\mathcal{V}(q, \bar{v})=\mathcal{U}_{1}(q, \bar{v}) c_{\theta}^{2}+\mathcal{U}_{3}(q, \bar{v}) s_{\theta}^{2}$, consequently, when $\mathcal{V}(q, \bar{v})=0$ the equations of motion (3.15) are satisfied identically, which corresponds to the propagation of non-dispersive waves. The secular equation (2.9) may then be used to obtain the wave speeds associated with $\mathcal{V}(q, \bar{v})=0$, which yields

$$
\bar{v}^{2}=c_{5} \quad \text { or } \quad \bar{v}^{2}=\frac{f_{1}^{(0)}}{\gamma_{21}-\gamma_{23}} .
$$

The implication is that the wave speed limit given by $\bar{v}_{0}^{2}=\mathcal{F}_{c}^{(0)} c_{\theta}^{2}+\mathcal{F}_{s}^{(0)} s_{\theta}^{2}$ is the only dispersive limit, whereas the two other limits, given by (3.13), and more explicitly by (3.16), are spurious limits associated with non-dispersive waves.

\section{(c) Second order}

In view of the equalities (3.10)-(3.12), the second order term of the dispersion relation (3.8) (adjusted to reflect the effect of use of (3.10)-(3.12) at leading order) may also 
be factorized, yielding

$$
\begin{aligned}
& \gamma_{21} \gamma_{23}\left(\left(\gamma_{23}-\gamma_{21}\right)\left(\gamma_{23} g_{1}^{2} c_{\theta}^{2}+\gamma_{21} g_{3}^{2} s_{\theta}^{2}-\gamma_{21} \gamma_{23} c_{4}\right)-\gamma_{21} \gamma_{23} f_{1}^{(0)}\right) \\
& \quad \times\left(q_{1}^{2}-q_{2}^{2}\right)\left(q_{1}^{2}-q_{3}^{2}\right)\left(q_{2}^{2}-q_{3}^{2}\right)\left(\bar{v}_{0}^{2}-c_{5}\right)\left(\bar{v}_{2}^{2}+\mathcal{F}_{c c}^{(2)} c_{\theta}^{4}+\mathcal{F}_{c s}^{(2)} s_{\theta}^{2} c_{\theta}^{2}+\mathcal{F}_{s s}^{(2)} s_{\theta}^{4}\right)=0,
\end{aligned}
$$

in which

$$
\begin{aligned}
& \mathcal{F}_{c c}^{(2)}=\frac{1}{3} \frac{\left(\mathcal{F}_{c}^{(0)}-2 \beta_{12}-2 g_{1}\right) g_{1}^{2}}{\gamma_{21}^{2}}, \quad \mathcal{F}_{s s}^{(2)}=\frac{1}{3} \frac{\left(\mathcal{F}_{s}^{(0)}-2 \beta_{23}-2 g_{3}\right) g_{3}^{2}}{\gamma_{23}^{2}}, \\
& \mathcal{F}_{c s}^{(2)}=\frac{1}{3}\left\{\frac{\left(\mathcal{F}_{c}^{(0)}-\gamma_{13}\right) g_{3}^{2}}{\gamma_{23}^{2}}-\frac{2 g_{1} g_{3}\left(g_{1}+g_{3}-\mu_{13}\right)}{\gamma_{21} \gamma_{23}}+\frac{\left(\mathcal{F}_{s}^{(0)}-\gamma_{31}\right) g_{1}^{2}}{\gamma_{21}^{2}}\right\} .
\end{aligned}
$$

It is then deduced that $\bar{v}_{2}^{2}=-\mathcal{F}_{c c}^{(2)} c_{\theta}^{4}-\mathcal{F}_{c s}^{(2)} c_{\theta}^{2} s_{\theta}^{2}-\mathcal{F}_{s s}^{(2)} s_{\theta}^{4}$ and the second order expansion for the square of scaled wave speed may be written as

$$
\bar{v}^{2}=\mathcal{F}_{s}^{(0)} s_{\theta}^{2}+\mathcal{F}_{c}^{(0)} c_{\theta}^{2}-\left(\mathcal{F}_{c c}^{(2)} c_{\theta}^{4}+\mathcal{F}_{c s}^{(2)} c_{\theta}^{2} s_{\theta}^{2}+\mathcal{F}_{s s}^{(2)} s_{\theta}^{4}\right) \eta^{2}+O\left(\eta^{4}\right) .
$$

(d) Relative asymptotic orders of displacements

The comparison of the relative orders of displacements and pressure increment is essential for the derivation of any lower dimensional model for long wave low frequency motion. Provided the dispersion relation (3.6) is satisfied, the homogeneous system of boundary conditions (3.5) possesses a non-trivial solution. Therefore, we may represent the coefficients $U_{2}^{(m)}, m \in\{1,2,3\}$, in terms of the single (constant with respect to $x_{2}$ ) parameter $U_{2}^{(0)}$

$$
\begin{aligned}
U_{2}^{(k)}= & (-1)^{k} \frac{\left(q_{i}^{2}-q_{j}^{2}\right) \mathcal{H}\left(q_{i}, q_{j}, \bar{v}\right) \mathcal{V}\left(q_{k}, \bar{v}\right)}{C_{k}(h)} U_{2}^{(0)}, \\
& i<j, \quad k \notin\{i, j\}, \quad i, j, k \in\{1,2,3\} .
\end{aligned}
$$

We previously remarked, that in the long wave low frequency regime, $q_{m}, m \in\{1,2,3\}$, is $O(1)$ as $\eta \rightarrow 0$. Hence, we may estimate the trigonometric functions occurring in (2.12) and (3.21) with the following expansions

$$
\begin{aligned}
S_{m}\left(x_{2}\right) & =q_{m} \frac{x_{2}}{h} \eta+O\left(\eta^{3}\right), \quad C_{m}\left(x_{2}\right)=1+O\left(\eta^{2}\right), \\
C_{m}(h) & =1+O\left(\eta^{2}\right), \quad m \in\{1,2,3\},
\end{aligned}
$$

in which we tacitly assume that $x_{2} / h \sim O(1)$. It is now possible to utilize relations (3.10)(3.12), as well as expansions (3.22), in order to determine the relative orders of displacements (2.12), yielding

$$
u_{1} \sim O\left(p_{t}\right), \quad u_{2} \sim \eta^{-1} O\left(p_{t}\right), \quad u_{3} \sim O\left(p_{t}\right),
$$


indicating that for this type of motion the leading order displacement is normal to the plane of the layer.

\section{Asymptotically approximate equations}

The derivation of asymptotically approximate equations involves the rescaling of space and time variables. The rescaling of space coordinates aims to balance the plate half-thickness $h$ and normal coordinate with a typical wave length $l$ and in-plane coordinates, so that we choose

$$
x_{1}=l \xi_{1}, \quad x_{2}=h \zeta=l \eta \zeta, \quad x_{3}=l \xi_{3},
$$

where $\xi_{1}, \zeta$ and $\xi_{3}$ are the new non-dimensional space coordinates. A typical wave of the considered type propagates with a scaled speed $\sqrt{\mathcal{F}^{(0)}}$, where $\mathcal{F}^{(0)} \equiv \mathcal{F}_{s}^{(0)} s_{\theta}^{2}+\mathcal{F}_{c}^{(0)} c_{\theta}^{2}$, see expansion (3.20), and thus travels the distance of a typical wave length $l$ in time $l \sqrt{\rho / \mathcal{F}^{(0)}}$ and the appropriate scale for time may be chosen in form

$$
t=\frac{l \sqrt{\rho}}{\sqrt{\mathcal{F}^{(0)}}} \tau,
$$

with $\tau$ denoting non-dimensional time. The distribution of the relative orders of displacements (3.23) dictates the asymptotic structure of the displacement components and pressure, motivating the following rescalings

$$
\begin{gathered}
u_{1}\left(\xi_{1}, \zeta, \xi_{3}, \tau\right)=l \eta u_{1}^{*}\left(\xi_{1}, \zeta, \xi_{3}, \tau\right), \quad u_{3}\left(\xi_{1}, \zeta, \xi_{3}, \tau\right)=l \eta u_{3}^{*}\left(\xi_{1}, \zeta, \xi_{3}, \tau\right), \\
u_{2}\left(\xi_{1}, \zeta, \xi_{3}, \tau\right)=l u_{2}^{*}\left(\xi_{1}, \zeta, \xi_{3}, \tau\right), \quad p_{t}\left(\xi_{1}, \zeta, \xi_{3}, \tau\right)=\eta \mathcal{F}^{(0)} p_{t}^{*}\left(\xi_{1}, \zeta, \xi_{3}, \tau\right),
\end{gathered}
$$

in which superscript * denotes non-dimensional quantities of a same asymptotic order.

We may now reformulate the equations of motion (2.5)-(2.7) in terms of the rescaled non-dimensional variables, so that

$$
\begin{gathered}
\gamma_{21} u_{1, \zeta \zeta}^{*}+\left(B_{1122}+B_{1221}\right) u_{2, \zeta \xi_{1}}^{*}+\eta^{2}\left\{B_{1111} u_{1, \xi_{1} \xi_{1}}^{*}\right. \\
\left.+\left(B_{1133}+B_{1331}\right) u_{3, \xi_{1} \xi_{3}}^{*}+\gamma_{31} u_{1, \xi_{3} \xi_{3}}^{*}-\mathcal{F}^{(0)}\left(u_{1, \tau \tau}^{*}+p_{t, \xi_{1}}^{*}\right)\right\}=0, \\
B_{2222} u_{2, \zeta \zeta}^{*}+\eta^{2}\left\{\gamma_{12} u_{2, \xi_{1} \xi_{1}}^{*}+\left(B_{1122}+B_{1221}\right) u_{1, \zeta \xi_{1}}^{*}\right. \\
\left.+\left(B_{2233}+B_{2332}\right) u_{3, \zeta \xi_{3}}^{*}+\gamma_{32} u_{2, \xi_{3} \xi_{3}}^{*}-\mathcal{F}^{(0)}\left(u_{2, \tau \tau}^{*}+p_{t, \zeta}^{*}\right)\right\}=0, \\
\gamma_{23} u_{3, \zeta \zeta}^{*}+\left(B_{2233}+B_{2332}\right) u_{2, \zeta \xi_{3}}^{*}+\eta^{2}\left\{B_{3333} u_{3, \xi_{3} \xi_{3}}^{*}\right. \\
\left.+\left(B_{1133}+B_{1331}\right) u_{1, \xi_{1} \xi_{3}}^{*}+\gamma_{13} u_{3, \xi_{1} \xi_{1}}^{*}-\mathcal{F}^{(0)}\left(u_{3, \tau \tau}^{*}+p_{t, \xi_{3}}^{*}\right)\right\}=0,
\end{gathered}
$$


in which a comma subscript denotes differentiation with respect to the indicated (space or time) variable. Equations (4.4)-(4.6) must be solved in conjunction with the appropriately rescaled incompressibility condition

$$
u_{2, \zeta}^{*}+\eta^{2}\left(u_{1, \xi_{1}}^{*}+u_{3, \xi_{3}}^{*}\right)=0
$$

and subject to the corresponding zero surface traction boundary conditions

$$
\begin{gathered}
\gamma_{21} u_{1, \zeta}^{*}+\left(B_{1221}+\bar{p}\right) u_{2, \xi_{1}}^{*}=0 \quad \text { at } \quad \zeta= \pm 1 \\
\left(B_{2222}+\bar{p}\right) u_{2, \zeta}^{*}+\eta^{2}\left(B_{1122} u_{1, \xi_{1}}^{*}+B_{2233} u_{3, \xi_{3}}^{*}-\mathcal{F}^{(0)} p_{t}^{*}\right)=0 \quad \text { at } \quad \zeta= \pm 1 \\
\gamma_{23} u_{3, \zeta}^{*}+\left(B_{2332}+\bar{p}\right) u_{2, \xi_{3}}^{*}=0 \quad \text { at } \quad \zeta= \pm 1 .
\end{gathered}
$$

All information regarding long wave low frequency contributions of different terms in equations (4.4)-(4.10) is now indicated explicitly through the appearance of $\eta^{2}$. This enables us to set up a perturbation scheme to obtain lower-dimensional governing equations for this type of motion. The solutions will be sought in a form of the power series

$$
\left(u_{1}^{*}, u_{2}^{*}, u_{3}^{*}, p_{t}^{*}\right)=\sum_{n=0}^{m} \eta^{2 n}\left(u_{1}^{*(2 n)}, u_{2}^{*(2 n)}, u_{3}^{*(2 n)}, p_{t}^{*(2 n)}\right)+O\left(\eta^{2 m+2}\right) .
$$

Substituting the solution (4.11) into the problem indicated by (4.4)-(4.10) we obtain a hierarchical system at various orders.

\section{(a) Leading order problem}

At leading order, the equations of motion (4.4)-(4.6) take the form

$$
\begin{gathered}
\gamma_{21} u_{1, \zeta \zeta}^{*(0)}+\left(B_{1122}+B_{1221}\right) u_{2, \zeta \xi_{1}}^{*(0)}=0 \\
B_{2222} u_{2, \zeta \zeta}^{*(0)}=0 \\
\gamma_{23} u_{3, \zeta \zeta}^{*(0)}+\left(B_{2233}+B_{2332}\right) u_{2, \zeta \xi_{3}}^{*(0)}=0
\end{gathered}
$$

which are to be solved subject to the leading order incompressibility condition

$$
u_{2, \zeta}^{*(0)}=0
$$

and appropriate leading order boundary conditions

$$
\gamma_{21} u_{1, \zeta}^{*(0)}+\left(B_{1221}+\bar{p}\right) u_{2, \xi_{1}}^{*(0)}=0 \quad \text { at } \quad \zeta= \pm 1
$$

Article submitted to Royal Society 


$$
\begin{gathered}
\left(B_{2222}+\bar{p}\right) u_{2, \zeta}^{*(0)}=0 \quad \text { at } \quad \zeta= \pm 1, \\
\gamma_{23} u_{3, \zeta}^{*(0)}+\left(B_{2332}+\bar{p}\right) u_{2, \xi_{3}}^{*(0)}=0 \quad \text { at } \quad \zeta= \pm 1 .
\end{gathered}
$$

The general solution of the boundary value problem (4.13), (4.17) may be represented as

$$
u_{2}^{*(0)}=U_{2}^{*(0,0)}
$$

in which $U_{2}^{*(0,0)}=U_{2}^{*(0,0)}\left(\xi_{1}, \xi_{3}, \tau\right)$. Henceforth, we use double superscripts to denote functions independent of $\zeta$, with the first superscript referring to the approximation order and second denoting the power of any possible $\zeta^{m}$ multiplier. Also note that our choice of scaling parameters ensures that the displacement components and pressure vary appropriately for flexural motion, i.e. as an anti-symmetric $\left(u_{1}^{*}, u_{3}^{*}\right.$ and $\left.p_{t}^{*}\right)$ or symmetric $\left(u_{2}^{*}\right)$ function of the normal coordinate $\zeta$. Thus, for the sake of brevity, we will always omit terms of the solutions which do not comply to this requirement.

Substituting the solution (4.19) into the boundary value problem (4.12), (4.16) it is possible to obtain the solution for $u_{1}^{*(0)}$ in the form

$$
u_{1}^{*(0)}=\zeta U_{1}^{*(0,1)}, \quad U_{1}^{*(0,1)}=-\frac{g_{1}}{\gamma_{21}} U_{2, \xi_{1}}^{*(0,0)}
$$

where we recall that $g_{m}=\gamma_{2 m}-\sigma_{2}, m \in\{1,3\}$. This procedure may be repeated for the problem (4.14), (4.18), to obtain

$$
u_{3}^{*(0)}=\zeta U_{3}^{*(0,1)}, \quad U_{3}^{*(0,1)}=-\frac{g_{3}}{\gamma_{23}} U_{2, \xi_{3}}^{*(0,0)} .
$$

We have now obtained leading order solutions for the displacement components in terms of the function $U_{2}^{*(0,0)}$, or more specifically, as linear functions of it and its derivatives. We will term the leading order normal displacement $U_{2}^{*(0,0)}$ as the leading order mid-surface deflection. Knowledge of this function determines the long wave low frequency motion at leading order. However, this function itself (and the leading order pressure increment) can not be determined without resorting to higher order.

\section{(b) Second order problem}

The second order problem is comprised of the appropriate equations of motion

$$
\begin{aligned}
& \gamma_{21} u_{1, \zeta \zeta}^{*(2)}+\left(B_{1122}+B_{1221}\right) u_{2, \zeta \xi_{1}}^{*(2)}= \\
& \quad-B_{1111} u_{1, \xi_{1} \xi_{1}}^{*(0)}-\left(B_{1133}+B_{1331}\right) u_{3, \xi_{1} \xi_{3}}^{*(0)}-\gamma_{31} u_{1, \xi_{3} \xi_{3}}^{*(0)}+\mathcal{F}^{(0)}\left(u_{1, \tau \tau}^{*(0)}+p_{t, \xi_{1}}^{*(0)}\right),
\end{aligned}
$$




$$
\begin{aligned}
& \mathcal{F}^{(0)} p_{t, \zeta}^{*(0)}=B_{2222} u_{2, \zeta \zeta}^{*(2)}+\gamma_{12} u_{2, \xi_{1} \xi_{1}}^{*(0)}+\gamma_{32} u_{2, \xi_{3} \xi_{3}}^{*(0)} \\
& +\left(B_{1122}+B_{1221}\right) u_{1, \xi_{1} \zeta}^{*(0)}+\left(B_{2233}+B_{2332}\right) u_{3, \zeta \xi_{3}}^{*(0)}-\mathcal{F}^{(0)} u_{2, \tau \tau}^{*(0)}, \\
& \gamma_{23} u_{3, \zeta \zeta}^{*(2)}+\left(B_{2233}+B_{2332}\right) u_{2, \zeta \xi_{3}}^{*(2)}= \\
& -\gamma_{13} u_{3, \xi_{1} \xi_{1}}^{*(0)}-\left(B_{1133}+B_{1331}\right) u_{1, \xi_{1} \xi_{3}}^{*(0)}-B_{3333} u_{3, \xi_{3} \xi_{3}}^{*(0)}+\mathcal{F}^{(0)}\left(u_{3, \tau \tau}^{*(0)}+p_{t, \xi_{3}}^{*(0)}\right),
\end{aligned}
$$

together with the second order incompressibility condition

$$
u_{2, \zeta}^{*(2)}=-u_{1, \xi_{1}}^{*(0)}-u_{3, \xi_{3}}^{*(0)}
$$

solved subject to the second order boundary conditions

$$
\begin{gathered}
\gamma_{21} u_{1, \zeta}^{*(2)}+\left(B_{1221}+\bar{p}\right) u_{2, \xi_{1}}^{*(2)}=0 \quad \text { at } \quad \zeta= \pm 1 \\
\mathcal{F}^{(0)} p_{t}^{*(0)}=\left(B_{2222}+\bar{p}\right) u_{2, \zeta}^{*(2)}+B_{1122} u_{1, \xi_{1}}^{*(0)}+B_{2233} u_{3, \xi_{3}}^{*(0)} \quad \text { at } \quad \zeta= \pm 1, \\
\gamma_{23} u_{3, \zeta}^{*(2)}+\left(B_{2332}+\bar{p}\right) u_{2, \xi_{3}}^{*(2)}=0 \quad \text { at } \quad \zeta= \pm 1 .
\end{gathered}
$$

Note, the leading order pressure $p_{t}^{*(0)}$ was deliberately shown on the left hand side of equations (4.23) and (4.27) to signify that it can only be determined at the current order.

Upon use of the leading order solutions (4.20) and (4.21), the incompressibility condition (4.25) may be utilized to establish the second order term of normal displacement

$$
u_{2}^{*(2)}=\zeta^{2} U_{2}^{*(2,2)}+U_{2}^{*(2,0)}, \quad U_{2}^{*(2,2)}=\frac{1}{2}\left\{\frac{g_{1}}{\gamma_{21}} U_{2, \xi_{1} \xi_{1}}^{*(0,0)}+\frac{g_{3}}{\gamma_{23}} U_{2, \xi_{3} \xi_{3}}^{*(0,0)}\right\},
$$

within which the function $U_{2}^{*(2,0)}$ can only be determined at third order. In view of (4.29), the analysis of the boundary value problem $(4.23),(4.27)$ reveals that it may only be satisfied by a solution of the following form

$$
p_{t}^{*(0)}=\zeta P_{t}^{*(1,0)}, \quad \mathcal{F}^{(0)} P_{t}^{*(1,0)}=\frac{g_{1}\left(b_{21}+g_{1}\right)}{\gamma_{21}} U_{2, \xi_{1} \xi_{1}}^{*(0,0)}+\frac{g_{3}\left(b_{23}+g_{3}\right)}{\gamma_{23}} U_{2, \xi_{3} \xi_{3}}^{*(0,0)},
$$

provided

$$
\mathcal{F}_{c}^{(0)} U_{2, \xi_{1} \xi_{1}}^{*(0,0)}+\mathcal{F}_{s}^{(0)} U_{2, \xi_{3} \xi_{3}}^{*(0,0)}-\mathcal{F}^{(0)} U_{2, \tau \tau}^{*(0,0)}=0
$$

with the functions of pre-stress and material parameters $\mathcal{F}_{c}^{(0)}$ and $\mathcal{F}_{s}^{(0)}$ defined in (3.14). The condition (4.31) was introduced to accommodate the second equation of motion (4.23) and provides the means to determine the leading order mid-surface deflection $U_{2}^{*(0,0)}$, hence we 
will refer to it as to the leading order governing equation for the mid-surface deflection. Since all leading order displacement components and incremental pressure are linear functions of $U_{2}^{*(0,0)}$ and its derivatives, equation $(4.31)$ will in fact be valid for all of $u_{1}^{*(0)}, u_{2}^{*(0)}, u_{3}^{*(0)}$ and $p_{t}^{*(0)}$, in particular we note that

$$
\mathcal{F}^{(0)} u_{m, \tau \tau}^{*(0)}=\mathcal{F}_{c}^{(0)} u_{m, \xi_{1} \xi_{1}}^{*(0)}+\mathcal{F}_{s}^{(0)} u_{m, \xi_{3} \xi_{3}}^{*(0)}, \quad m \in\{1,3\}
$$

Using (4.32) to dispose of time derivatives in the first equation of motion (4.22), it is possible to conclude that the second order displacement coefficient $u_{1}^{*(2)}$ has the form

$$
u_{1}^{*(2)}=\zeta^{3} U_{1}^{*(2,3)}+\zeta U_{1}^{*(2,1)},
$$

which, after inserting this into the first equation of motion (4.22) and the first boundary condition (4.26), yields

$$
\begin{aligned}
& U_{1}^{*(2,3)}=-\frac{1}{6}\left\{\left(\frac{g_{1}^{2}}{\gamma_{21}^{2}}+f_{2,13}\right) U_{2, \xi_{1} \xi_{1} \xi_{1}}^{*(0,0)}+\left(\frac{g_{1} g_{3}}{\gamma_{21} \gamma_{23}}+f_{2,11}\right) U_{2, \xi_{1} \xi_{3} \xi_{3}}^{*(0,0)}\right\}, \\
& U_{1}^{*(2,1)}=-\frac{g_{1}}{\gamma_{21}} U_{2, \xi_{1}}^{*(2,0)}+\frac{1}{2}\left\{f_{13}^{(2)} U_{2, \xi_{1} \xi_{1} \xi_{1}}^{*(0,0)}+f_{11}^{(2)} U_{2, \xi_{1} \xi_{3} \xi_{3}}^{*(0,0)}\right\}
\end{aligned}
$$

where

$$
f_{13}^{(2)}=\frac{3 \mathcal{F}_{c c}^{(2)}}{g_{1}}, \quad f_{11}^{(2)}=\frac{g_{1}\left(\mathcal{F}_{s}^{(0)}-\gamma_{31}\right)}{\gamma_{21}^{2}}+\frac{g_{3}\left(\mu_{13}-g_{1}-g_{3}\right)}{\gamma_{21} \gamma_{23}},
$$

with the function of the material parameters and pre-stress $\mathcal{F}_{c c}^{(2)}$ defined immediately after (3.17). The same mathematical manipulation sequence repeated for the boundary value problem (4.22), (4.26) delivers the second order solution for the third displacement component, given by

$$
\begin{gathered}
u_{3}^{*(2)}=\zeta^{3} U_{3}^{*(2,3)}+\zeta U_{3}^{*(2,1)}, \\
U_{3}^{*(2,3)}=-\frac{1}{6}\left\{\left(\frac{g_{1} g_{3}}{\gamma_{21} \gamma_{23}}+f_{31}^{(2)}\right) U_{2, \xi_{1} \xi_{1} \xi_{3}}^{*(0,0)}+\left(\frac{g_{3}^{2}}{\gamma_{23}^{2}}+f_{33}^{(2)}\right) U_{2, \xi_{3} \xi_{3} \xi_{3}}^{*(0,0)}\right\}, \\
U_{3}^{*(2,1)}=-\frac{g_{3}}{\gamma_{23}} U_{2, \xi_{3}}^{*(2,0)}+\frac{1}{2}\left\{f_{31}^{(2)} U_{2, \xi_{1} \xi_{1} \xi_{3}}^{*(0,0)}+f_{33}^{(2)} U_{2, \xi_{3} \xi_{3} \xi_{3}}^{*(0,0)}\right\},
\end{gathered}
$$

in which

$$
f_{31}^{(2)}=\frac{g_{3}\left(\mathcal{F}_{c}^{(0)}-\gamma_{13}\right)}{\gamma_{23}^{2}}+\frac{g_{1}\left(\mu_{13}-g_{1}-g_{3}\right)}{\gamma_{21} \gamma_{23}}, \quad f_{33}^{(2)}=\frac{3 \mathcal{F}_{s s}^{(2)}}{g_{3}},
$$

with the parameter $\mathcal{F}_{s s}^{(2)}$ defined immediately after (3.17).

We shall now reconsider the leading order equation for a mid-surface deflection (4.31), which in terms of the original variables may be recast as

$$
\mathcal{F}_{c}^{(0)} \frac{\partial^{2} u_{0}}{\partial x_{1}^{2}}+\mathcal{F}_{s}^{(0)} \frac{\partial^{2} u_{0}}{\partial x_{3}^{2}}-\rho \frac{\partial^{2} u_{0}}{\partial t^{2}}=0
$$

Article submitted to Royal Society 
in which we introduced $u_{0}\left(x_{1}, x_{3}, t\right) \equiv U_{2}^{*(0,0)}\left(\xi_{1}, \xi_{3}, \tau\right)$. It was mentioned previously that the solution of equation (4.37) enables the leading order solutions for all displacement components and pressure to be determined. Also, the dispersion relation associated with this equation is precisely the leading order approximation for wave speed given immediately after (3.14), which demonstrates the asymptotic consistency of the model. It is interesting to note that the dispersion relation associated with the leading order governing equations for long wave high frequency motion, or motion in the vicinity of the shear resonance frequencies, matches the second order dispersion relation approximation, see Pichugin \& Rogerson (2001a). However, in the present case the second order pressure $p_{t, \zeta}^{*(2)}$ can only be obtained at third order, in terms of the leading order mid-surface deflection and its second order correction, thus the satisfying of the equation (4.37) does not guarantee the consistency of the model at second order.

\section{(c) Third order problem}

Instead of solving all of the third order equations, we only consider those necessary to complete the second order solution and obtain a second order governing equation. Hence, we examine the second equation of motion

$$
\begin{aligned}
& \mathcal{F}^{(0)} p_{t, \zeta}^{*(2)}=B_{2222} u_{2, \zeta \zeta}^{*(4)}+\gamma_{12} u_{2, \xi_{1} \xi_{1}}^{*(2)} \\
& \quad+\left(B_{1122}+B_{1221}\right) u_{1, \zeta \xi_{1}}^{*(2)}+\left(B_{2233}+B_{2332}\right) u_{3, \zeta \xi_{3}}^{*(2)}+\gamma_{32} u_{2, \xi_{3} \xi_{3}}^{*(2)}-\mathcal{F}^{(0)} u_{2, \tau \tau}^{*(2)}
\end{aligned}
$$

subject to the third order incompressibility condition

$$
u_{2, \zeta}^{*(4)}=-u_{1, \xi_{1}}^{*(2)}-u_{3, \xi_{3}}^{*(2)}
$$

and the third order form of the second boundary condition, namely

$$
\mathcal{F}^{(0)} p_{t}^{*(2)}=\left(B_{2222}+\bar{p}\right) u_{2, \zeta}^{*(4)}+B_{1122} u_{1, \xi_{1}}^{*(2)}+B_{2233} u_{3, \xi_{3}}^{*(2)} \quad \text { at } \quad \zeta= \pm 1
$$

Inserting the solutions (4.33) and (4.35) into the incompressibility condition (4.39) it is possible to deduce a general solution for $u_{2}^{*(4)}$ in the form

$$
u_{2}^{*(4)}=\zeta^{4} U_{2}^{*(4,4)}+\zeta^{2} U_{2}^{*(4,2)}+U_{2}^{*(4,0)},
$$

Article submitted to Royal Society 
which when coupled with the boundary condition (4.40), yields

$$
\begin{aligned}
U_{2}^{*(4,2)}= & \frac{1}{2}\left\{\frac{g_{1}}{\gamma_{21}} U_{2, \xi_{1} \xi_{1}}^{*(2,0)}+\frac{g_{3}}{\gamma_{23}} U_{2, \xi_{3} \xi_{3}}^{*(2,0)}\right\} \\
- & \frac{1}{4}\left\{f_{13}^{(2)} U_{2, \xi_{1} \xi_{1} \xi_{1} \xi_{1}}^{*(0,0)}+\left(f_{11}^{(2)}+f_{31}^{(2)}\right) U_{2, \xi_{1} \xi_{1} \xi_{3} \xi_{3}}^{*(0,0)}+f_{33}^{(2)} U_{2, \xi_{3} \xi_{3} \xi_{3} \xi_{3}}^{*(0,0)}\right\}, \\
U_{2}^{*(4,4)} & =\frac{1}{24}\left\{\left(2 \frac{g_{1} g_{3}}{\gamma_{21} \gamma_{23}}+f_{11}^{(2)}+f_{31}^{(2)}\right) U_{2, \xi_{1} \xi_{1} \xi_{3} \xi_{3}}^{*(0,0)}\right. \\
+ & \left(\frac{g_{1}^{2}}{\gamma_{21}^{2}}+f_{13}^{(2)}\right) U_{2, \xi_{1} \xi_{1} \xi_{1} \xi_{1}}^{*(0,0)}+\left(\frac{g_{3}^{2}}{\gamma_{23}^{2}}+f_{33}^{(2)}\right) U_{2, \xi_{3} \xi_{3} \xi_{3} \xi_{3}}^{*}(0,0)
\end{aligned}
$$

and $U_{2}^{*(4,0)}$ can only be determined at the higher order. The equation of motion (4.38) may then be used to establish a general solution for $p_{t}^{*(2)}$, given by

$$
p_{t}^{*(2)}=\zeta^{3} P_{t}^{*(3,2)}+\zeta P_{t}^{*(1,2)},
$$

where

$$
\begin{aligned}
\mathcal{F}^{(0)} P_{t}^{*(3,2)} & =\frac{1}{6}\left\{\left(b_{21}\left(\frac{g_{1}^{2}}{\gamma_{21}^{2}}+f_{13}^{(2)}\right)+\frac{g_{1}}{\gamma_{21}}\left(\gamma_{12}-\mathcal{F}_{c}^{(0)}\right)\right) U_{2, \xi_{1} \xi_{1} \xi_{1} \xi_{1}}^{*(0,0)}+\left\{\frac{g_{1}}{\gamma_{21}}\left(\gamma_{32}-\mathcal{F}_{s}^{(0)}\right)\right.\right. \\
& \left.+b_{21}\left(\frac{g_{1} g_{3}}{\gamma_{21} \gamma_{23}}+f_{11}^{(2)}\right)+b_{23}\left(\frac{g_{1} g_{3}}{\gamma_{21} \gamma_{23}}+f_{31}^{(2)}\right)+\frac{g_{3}}{\gamma_{23}}\left(\gamma_{12}-\mathcal{F}_{c}^{(0)}\right)\right\} U_{2, \xi_{1} \xi_{1} \xi_{3} \xi_{3}}^{*(0,0)} \\
& \left.+\left(b_{23}\left(\frac{g_{3}^{2}}{\gamma_{23}^{2}}+f_{33}^{(2)}\right)+\frac{g_{3}}{\gamma_{23}}\left(\gamma_{32}-\mathcal{F}_{s}^{(0)}\right)\right) U_{2, \xi_{3} \xi_{3} \xi_{3} \xi_{3}}^{*(0,0)}\right\} .
\end{aligned}
$$

Finally, we use the boundary condition (4.40) to obtain

$$
\begin{aligned}
\mathcal{F}^{(0)} & P_{t}^{*(1,2)}=\frac{g_{1}\left(b_{21}+g_{1}\right)}{\gamma_{21}} U_{2, \xi_{1} \xi_{1}}^{*(2,0)}+\frac{g_{3}\left(b_{23}+g_{3}\right)}{\gamma_{23}} U_{2, \xi_{3} \xi_{3}}^{*(2,0)} \\
+ & \frac{1}{6}\left\{\left(\frac{g_{1}^{3}}{\gamma_{21}^{2}}-\frac{g_{1}}{\gamma_{21}}\left(\gamma_{12}-\mathcal{F}_{c}^{(0)}\right)-f_{13}^{(2)}\left(2 g_{1}+3 b_{21}\right)\right) U_{2, \xi_{1} \xi_{1} \xi_{1} \xi_{1}}^{*(0,0)}-\left\{\frac{g_{1}}{\gamma_{21}}\left(\gamma_{32}-\mathcal{F}_{s}^{(0)}\right)\right.\right. \\
& \left.-\frac{g_{1} g_{3}\left(g_{1}+g_{3}\right)}{\gamma_{21} \gamma_{23}}+\frac{g_{3}}{\gamma_{23}}\left(\gamma_{12}-\mathcal{F}_{c}^{(0)}\right)+f_{11}^{(2)}\left(2 g_{1}+3 b_{21}\right)+f_{31}^{(2)}\left(2 g_{3}+3 b_{23}\right)\right\} U_{2, \xi_{1} \xi_{1} \xi_{3} \xi_{3}}^{*(0,0)} \\
& \left.+\left(\frac{g_{3}^{3}}{\gamma_{23}^{2}}-\frac{g_{3}}{\gamma_{23}}\left(\gamma_{32}-\mathcal{F}_{s}^{(0)}\right)-f_{33}^{(2)}\left(2 g_{3}+3 b_{23}\right)\right) U_{2, \xi_{3} \xi_{3} \xi_{3} \xi_{3}}^{*(0,0)}\right\}
\end{aligned}
$$

which is only valid provided

$$
\begin{aligned}
\mathcal{F}_{c}^{(0)} U_{2, \xi_{1} \xi_{1}}^{*(2,0)}+\mathcal{F}_{s}^{(0)} U_{2, \xi_{3} \xi_{3}}^{*(2,0)}-\mathcal{F}^{(0)} U_{2, \tau \tau}^{*(2,0)} & \\
& +\mathcal{F}_{c c}^{(2)} U_{2, \xi_{1} \xi_{1} \xi_{1} \xi_{1}}^{*(0,0)}+\mathcal{F}_{c s}^{(2)} U_{2, \xi_{1} \xi_{1} \xi_{3} \xi_{3}}^{*(0,0)}+\mathcal{F}_{s s}^{(2)} U_{2, \xi_{3} \xi_{3} \xi_{3} \xi_{3}}^{*(0,0)}=0
\end{aligned}
$$

within which use has been made of the definitions (3.19), (4.34) and (4.36) to conclude that $\mathcal{F}_{c s}^{(2)}=\frac{1}{3}\left(g_{1} f_{11}^{(2)}+g_{3} f_{31}^{(2)}\right)$. Let us introduce a new function $u\left(x_{1}, x_{3}, t\right)=u^{*}\left(\xi_{1}, \xi_{3}, \tau\right)$ as

$$
u=u^{(0)}+u^{(2)} \eta^{2}+O\left(\eta^{4}\right), \quad u^{*}=u^{*(0)}+u^{*(2)} \eta^{2}+O\left(\eta^{4}\right),
$$


where we assume $u^{*(m)} \equiv U_{2}^{*(m, 0)}, m=1,2,3 \ldots$ Note, when $\zeta=0$ all of the solutions for $u_{1}^{*(m)}, u_{3}^{*(m)}$ and $p_{t}^{*(m)}, m \in\{0,2,4\}$, vanish, except the solutions for $u_{2}^{*(m)}$. This strongly supports the choice of the term mid-surface deflection for $u$. We also remark, that since every function $U_{2}^{*(2 m, 0)}$ satisfies each boundary value problem posed for $U_{2}^{*(0,0)}, \ldots U_{2}^{*(2 m-2,0)}$, then every displacement component and pressure may be represented as a linear function of $u$ and its derivatives, thus any governing equation for $u$ is also valid for all of the displacement components and pressure.

In view of the definition (4.44), the linear combination $(4.31)+\eta^{2}(4.43)$ gives the refined (second order) governing equation for a mid-surface deflection

$$
\mathcal{F}_{c}^{(0)} \frac{\partial^{2} u^{*}}{\partial \xi_{1}^{2}}+\mathcal{F}_{s}^{(0)} \frac{\partial^{2} u^{*}}{\partial \xi_{3}^{2}}-\mathcal{F}^{(0)} \frac{\partial^{2} u^{*}}{\partial \tau^{2}}+\eta^{2}\left(\mathcal{F}_{c c}^{(2)} \frac{\partial^{4} u^{*}}{\partial \xi_{1}^{4}}+\mathcal{F}_{c s}^{(2)} \frac{\partial^{4} u^{*}}{\partial \xi_{1}^{2} \partial \xi_{3}^{2}}+\mathcal{F}_{s s}^{(2)} \frac{\partial^{4} u^{*}}{\partial \xi_{3}^{4}}\right)=0
$$

which may also be written in terms of the non-scaled variables as

$$
\mathcal{F}_{c}^{(0)} \frac{\partial^{2} u}{\partial x_{1}^{2}}+\mathcal{F}_{s}^{(0)} \frac{\partial^{2} u}{\partial x_{3}^{2}}-\rho \frac{\partial^{2} u}{\partial t^{2}}+h^{2}\left(\mathcal{F}_{c c}^{(2)} \frac{\partial^{4} u}{\partial x_{1}^{4}}+\mathcal{F}_{c s}^{(2)} \frac{\partial^{4} u}{\partial x_{1}^{2} \partial x_{3}^{2}}+\mathcal{F}_{s s}^{(2)} \frac{\partial^{4} u}{\partial x_{3}^{4}}\right)=0
$$

The dispersion relation associated with equation (4.46) matches the second order wave speed approximation (3.20) exactly.

\section{(d) Analogy with an elastic membrane}

The leading order governing equation for the mid-surface deflection (4.37) is closely connected with the classic membrane equation. Indeed, in the absence of normal pre-stress $\left(\sigma_{2}=0\right) \bar{p}=\gamma_{21}-B_{1221}=\gamma_{23}-B_{2332}$, see (3.4). We may also specify the components of the in-plane Cauchy stresses as pure tensions

$$
\sigma_{1}=T_{1}, \quad \sigma_{3}=T_{3}
$$

which coupled with the relations (3.4) enables us to conclude that $\mathcal{F}_{c}^{(0)}=T_{1}$ and $\mathcal{F}_{s}^{(0)}=T_{3}$, thus recasting (4.37) as a membrane-like equation

$$
T_{1} \frac{\partial^{2} u^{(0)}}{\partial x_{1}^{2}}+T_{3} \frac{\partial^{2} u^{(0)}}{\partial x_{3}^{2}}-\rho \frac{\partial^{2} u^{(0)}}{\partial t^{2}}=0
$$

with $u^{(0)}$ playing role of the normal deflection of an infinite membrane. The second order governing equation may also be appropriately specialized, yielding the refined membrane 
equation

$$
\begin{gathered}
T_{1} \frac{\partial^{2} u}{\partial x_{1}^{2}}+T_{3} \frac{\partial^{2} u}{\partial x_{3}^{2}}-\rho \frac{\partial^{2} u}{\partial t^{2}}+\frac{h^{2}}{3}\left\{\left(T_{1}-2 \beta_{12}-2 \gamma_{21}\right) \frac{\partial^{4} u}{\partial x_{1}^{4}}+\left(T_{3}-2 \beta_{23}-2 \gamma_{23}\right) \frac{\partial^{4} u}{\partial x_{3}^{4}}\right. \\
\left.+\left(T_{1}-\gamma_{13}+T_{3}-\gamma_{31}+2\left(\mu_{13}-\gamma_{21}-\gamma_{23}\right)\right) \frac{\partial^{4} u}{\partial x_{1}^{2} \partial x_{3}^{2}}\right\}=0
\end{gathered}
$$

We remark that the coefficients in the refined membrane equation (4.49) involve both material parameters and thickness, in addition to tensions and material density. This equation is seemingly the first refined membrane equation derived without utilizing any ad hoc assumptions. This result complements Kaplunov et al. (2000) where a similar plain-strain model mimicked the equation for waves on an infinite string.

\section{Model problem: shock edge loading}

As an illustration of the theory, we shall now consider the problem of shock edge loading of a semi-infinite plate with free faces. In order to simplify the problem we focus attention to the case when the edge surface is orthogonal to the direction of wave propagation $\left(c_{\theta}, 0, s_{\theta}\right)$. It is then convenient to rotate the in-plane coordinate axes as follows

$$
\xi_{n}=c_{\theta} \xi_{1}+s_{\theta} \xi_{3}, \quad \xi_{t}=-s_{\theta} \xi_{1}+c_{\theta} \xi_{3}
$$

in which coordinates $\xi_{n}$ and $\xi_{t}$ are normal and tangential to the edge $\xi_{n}=0$, respectively. For the problem of homogeneous edge loading it is natural to assume no variation of displacement components and pressure with respect to $\xi_{t}$. Therefore, the coordinate transformation (5.1) enables the governing equation (4.45) to be expressed as

$$
\mathcal{F}^{(0)}\left(\frac{\partial^{2} u^{*}\left(\xi_{n}, \tau\right)}{\partial \xi_{n}^{2}}-\frac{\partial^{2} u^{*}\left(\xi_{n}, \tau\right)}{\partial \tau^{2}}\right)+\eta^{2} \mathcal{F}^{(2)} \frac{\partial^{4} u^{*}\left(\xi_{n}, \tau\right)}{\partial \xi_{n}^{4}}=0
$$

with $u^{*}\left(\xi_{n}, \tau\right) \equiv u^{*}\left(\xi_{1}, \xi_{3}, \tau\right)$ and $\mathcal{F}^{(2)} \equiv \mathcal{F}_{c c}^{(2)} c_{\theta}^{4}+\mathcal{F}_{c s}^{(2)} c_{\theta}^{2} s_{\theta}^{2}+\mathcal{F}_{s s}^{(2)} s_{\theta}^{4}$. A surface parallel to the edge of the plate is defined by the outward normal $\left(c_{\theta}, 0, s_{\theta}\right)$. Thus, $(2.4)$ gives the measure of incremental surface traction on any surface parallel to the edge in the component form

$$
\begin{aligned}
\tau_{\theta 1}= & \left(\left(B_{1111}+\bar{p}\right) u_{1,1}+B_{1122} u_{2,2}+B_{1133} u_{3,3}-p_{t}\right) c_{\theta}+\left(B_{3131} u_{1,3}+\left(B_{3113}+\bar{p}\right) u_{3,1}\right) s_{\theta}, \\
& \tau_{\theta 2}=\left(\left(B_{1221}+\bar{p}\right) u_{1,2}+B_{1212} u_{2,1}\right) c_{\theta}+\left(B_{3232} u_{2,3}+\left(B_{3223}+\bar{p}\right) u_{3,2}\right) s_{\theta} \\
\tau_{\theta 3}= & \left(\left(B_{1331}+\bar{p}\right) u_{1,3}+B_{1313} u_{3,1}\right) c_{\theta}+\left(B_{3311} u_{1,1}+B_{3322} u_{2,2}+\left(B_{3333}+\bar{p}\right) u_{3,3}-p_{t}\right) s_{\theta}
\end{aligned}
$$


in which $\tau_{\theta m}, m \in\{1,2,3\}$, is the projection of traction onto the appropriate coordinate axis. We may determine relative orders of traction components by substituting the leading order solutions for the displacement components (4.19)-(4.21) and pressure (4.30) into the expressions (5.3)-(5.5) to yield

$$
\tau_{\theta 1}^{*}=O(\eta), \quad \tau_{\theta 2}^{*}=\mathcal{F}_{c}^{(0)} U_{2, \xi_{1}}^{*(0,0)} c_{\theta}+\mathcal{F}_{s}^{(0)} U_{2, \xi_{3}}^{*(0,0)} s_{\theta}+O\left(\eta^{2}\right), \quad \tau_{\theta 3}^{*}=O(\eta)
$$

This result clearly indicates that in the long wave low frequency regime the component of traction $\tau_{\theta 2}^{*}$ is asymptotically leading. Therefore, we may utilize definitions (5.1) and (4.44) to construct the boundary condition for the governing equation (5.2) in the form

$$
\tau_{\theta 2}^{*}=\mathcal{F}^{(0)} \frac{\partial u^{*}\left(\xi_{n}, \tau\right)}{\partial \xi_{n}} \text { at } \xi_{n}=0
$$

where $\mathcal{F}^{(0)} \equiv \mathcal{F}_{c}^{(0)} c_{\theta}^{2}+\mathcal{F}_{s}^{(0)} s_{\theta}^{2}$. Note that the assumptions made in (5.7) imply a discrepancy of $O(\eta)$ in the traction components $\tau_{\theta 1}^{*}$ and $\tau_{\theta 3}^{*}$. This discrepancy may, for example, be tackled by considering quasi-static boundary layers along the edge, however this discussion is deferred for further study.

\section{(a) Shock edge loading}

The solution of the shock edge loading problem for the leading order governing equation (4.31) contains a discontinuity at the wave front. The fourth order derivative term in the singularly perturbed governing equation (4.45) smoothes this discontinuity. This phenomenon is known in the theory of thin structures as a quasi-front, see for example Kaplunov et al. (1998). The method of matched asymptotic expansions, see Cole (1968), provides one possible way of analysing this phenomenon. Let us consider the problem of a semi-infinite plate subject to the shock edge loading

$$
T^{*} \equiv \tau_{\theta 2}^{*}=M H(\tau) \quad \text { at } \quad \xi_{n}=0,
$$

in which $M$ is the magnitude of the load and $H(\tau)$ is the unit Heaviside function. The governing equation (5.2) is a classical wave equation perturbed by a term of $O\left(\eta^{2}\right)$, this term providing significant contribution only when $\xi_{n}$ varies rapidly. This is of particular importance within a thin boundary layer in the vicinity of quasi-front. In our approach, the solution of (5.2), termed the inner solution, is not subjected to the boundary condition (5.7). Instead, we first analyse the boundary value problem (4.31), (5.7), with appropriately standardized variables, whose solution will be referred to as outer solution, and then a matching 
condition between inner and outer solution compensates for the boundary condition required for the inner (boundary layer) problem.

The introduction of the inner (boundary layer) coordinates

$$
\xi_{\text {in }}=\eta^{-2 / 3}\left(\tau-\xi_{n}\right), \quad \tau_{i n}=\tau,
$$

is aimed at asymptotically balancing time and the fourth order space derivatives in the governing equation (5.2), which then takes the appropriate form

$$
2 \mathcal{F}^{(0)} \frac{\partial^{2} u^{*}\left(\xi_{i n}, \tau_{i n}\right)}{\partial \xi_{i n} \partial \tau_{i n}}-\mathcal{F}^{(2)} \frac{\partial^{4} u^{*}\left(\xi_{i n}, \tau_{i n}\right)}{\partial \xi_{i n}^{4}}=0
$$

The inner (boundary layer) traction $T_{i n}^{*}\left(\xi_{i n}, \tau_{i n}\right)$ may now be introduced, which upon use of $(5.6)_{2}$ recasts equation $(5.10)$ as

$$
\frac{\partial T_{i n}^{*}\left(\xi_{\text {in }}, \tau_{i n}\right)}{\partial \tau_{i n}}-\frac{1}{2} \frac{\mathcal{F}^{(2)}}{\mathcal{F}^{(0)}} \frac{\partial^{3} T_{i n}^{*}\left(\xi_{\text {in }}, \tau_{i n}\right)}{\partial \xi_{\text {in }}^{3}}=0,
$$

where $T_{i n}^{*}\left(\xi_{i n}, \tau_{i n}\right)=2 \mathcal{F}^{(0)} \partial u^{*}\left(\xi_{i n}, \tau_{i n}\right) / \partial \xi_{i n}$. Equation (5.11) may be solved using Fourier transforms to obtain the solution, which in terms of original scaled variables takes the form

$$
T_{i n}^{*}\left(\xi_{n}, \tau\right)=\frac{1}{2 \pi} \int_{-\infty}^{\infty} T_{F}\left(\eta^{2 / 3} \zeta\right) \exp \left(-i \frac{1}{2} \frac{\mathcal{F}^{(2)}}{\mathcal{F}(0)} \eta^{2} \zeta^{3} \tau\right) e^{i \zeta\left(\tau-\xi_{n}\right)} \eta^{2 / 3} d \zeta
$$

with the function $T_{F}\left(\eta^{2 / 3} \zeta\right)$ to be determined.

The outer problem is governed by the leading order governing equation (4.31), which in view of the coordinate rotation (5.1) and the normal traction component definition $(5.6)_{2}$ may be written as

$$
\frac{\partial^{2} T_{o u t}^{*}\left(\xi_{n}, \tau\right)}{\partial \xi_{n}{ }^{2}}-\frac{\partial^{2} T_{o u t}^{*}\left(\xi_{n}, \tau\right)}{\partial \tau^{2}}=0,
$$

and solved subject to the boundary condition (5.8) given explicitly as

$$
T_{\text {out }}^{*}(0, \tau)=M H(\tau)
$$

The boundary value problem $(5.13),(5.14)$ is a standard signalling problem for a semiinfinite interval, see e.g. Kevorkian (1989, p. 153). The appropriate solution is given by one of D'Alembert waves propagating away from the edge, and may upon applying the boundary condition (5.14) be expressed as

$$
T_{\text {out }}^{*}\left(\xi_{n}, \tau\right)=M H\left(\tau-\xi_{n}\right)=\frac{M}{2 \pi} \int_{-\infty}^{\infty}\left(\pi \delta(\zeta)-\frac{i}{\zeta}\right) e^{i \zeta\left(\tau-\xi_{n}\right)} d \zeta,
$$




\begin{tabular}{|c|c|c|c|c|}
\hline$\mu$ & $\lambda_{1}$ & $\lambda_{2}$ & $\sigma_{2}$ & $\eta$ \\
\hline 3.1 & 1.0 & 0.8 & 1.6 & 0.5 \\
\hline
\end{tabular}

Table 1. Parameters used in generating graphs.

with $\delta(\zeta)$ the Dirac delta function. It is remarked that the value of the improper integral on right-hand side of (5.15) is assumed to be the Cauchy principal value. We may now compare the definitions of the inner and outer traction $T_{i n}^{*}\left(\xi_{n}, \tau\right)$ and $T_{o u t}^{*}\left(\xi_{n}, \tau\right)$ to deduce that $\eta^{-2 / 3} T_{i n}^{*}\left(\xi_{n}, \tau\right)=-2 T_{\text {out }}^{*}\left(\xi_{n}, \tau\right)$. The matching procedure requires that the limit of $T_{i n}^{*}\left(\xi_{n}, \tau\right)$ as $\eta \rightarrow 0$ must be equal to $T_{\text {out }}^{*}\left(\xi_{n}, \tau\right)$, which gives

$$
T_{F}\left(\eta^{2 / 3} \zeta\right)=-2 M\left(\pi \delta(\zeta)-\frac{i}{\zeta}\right)
$$

Therefore, in view of (5.16) we may integrate (5.12) to obtain

$$
T^{*}\left(\xi_{n}, \tau\right)=\frac{M}{2}+\frac{M}{6} \operatorname{sgn} c_{\mathcal{F}}+M \int_{0}^{z_{0}} \operatorname{Ai}\left(z \operatorname{sgn} c_{\mathcal{F}}\right) d z,
$$

in which Ai is the Airy function and

$$
z_{0}=\frac{\tau-\xi_{n}}{\left|c_{\mathcal{F}}\right|^{1 / 3}}, \quad c_{\mathcal{F}}=-\frac{3}{2} \frac{\mathcal{F}^{(2)} \eta^{2} \tau}{\mathcal{F}^{(0)}} .
$$

(b) Typical wave profiles

In order to demonstrate the behaviour of the solution (5.17), plots of typical wave profiles were generated in respect of a Varga material, defined by the strain energy function

$$
W\left(\lambda_{1}, \lambda_{2}, \lambda_{3}\right)=\mu\left(\lambda_{1}+\lambda_{2}+\lambda_{3}-3\right)
$$

where $\mu$ is a shear modulus. All non-zero components of the elasticity tensor $B_{i j k l}$ are given in this case by

$$
B_{i j i j}=\frac{\mu \lambda_{i}^{2}}{\lambda_{i}+\lambda_{j}}, \quad B_{i j j i}=-\frac{\mu \lambda_{i} \lambda_{j}}{\lambda_{i}+\lambda_{j}}, \quad i \neq j, \quad i, j \in\{1,2,3\} .
$$

Table 1 shows the set of material parameters used to generate illustrations.

In contrast to the classic isotropic case, see for example Kaplunov et al. (1998), the sign of the third order derivative term in the governing equation (5.11) may change. When $\operatorname{sgn} c_{\mathcal{F}}<0$, the solution (5.17) generates a receding wave front, see figure 1 . When $\operatorname{sgn} c_{\mathcal{F}}>0$ however, the wave front is advancing, see figure 2 ; this latter case has no analog in the classical linear isotropic theory. Figures 1 and 2 also show that the type of quasi-front 

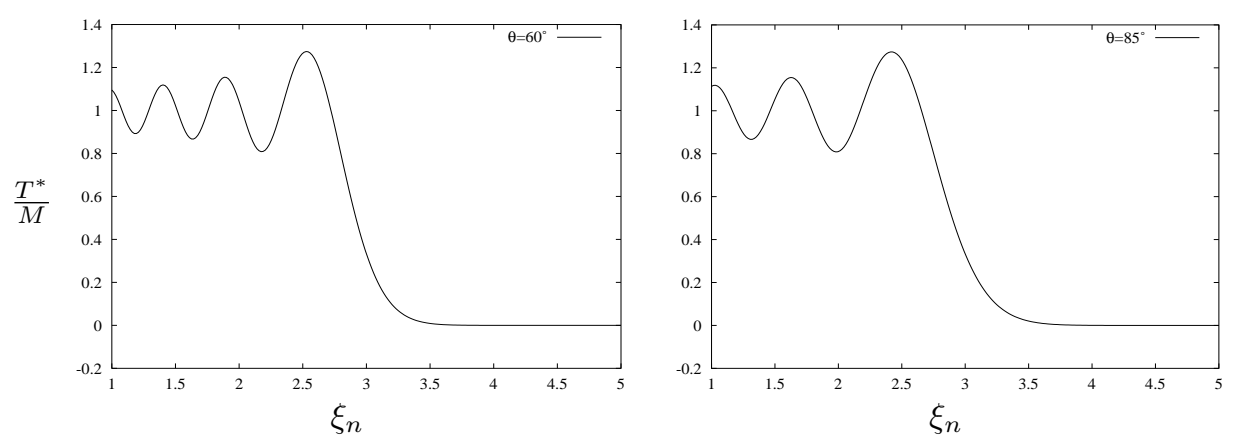

Figure 1. The receding wave front profiles at $\tau=3$, shown for the different angles $\theta$ of wave propagation. Table 1 summarizes utilized material parameters.
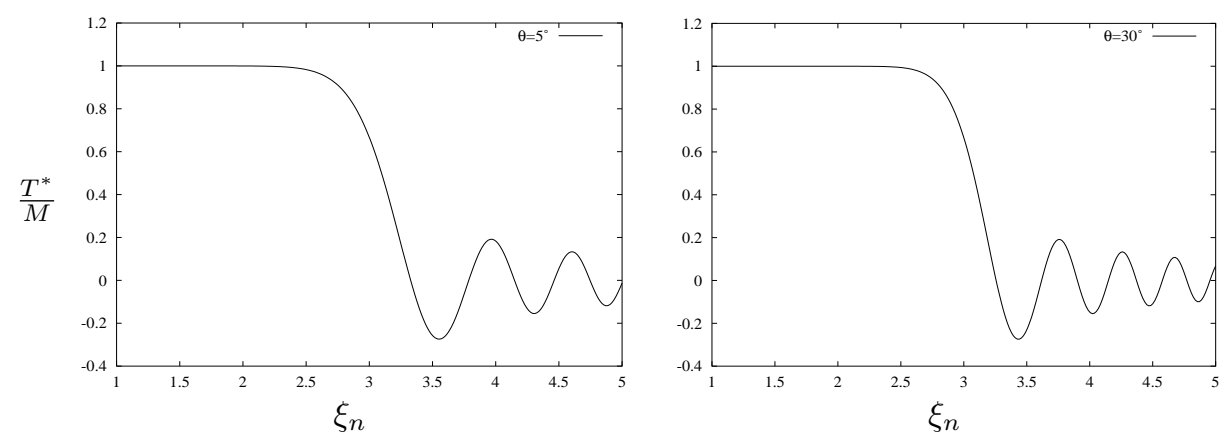

Figure 2. The advancing wave front profiles at $\tau=3$ shown for the different angles $\theta$ of wave propagation. Table 1 summarizes utilized material parameters.
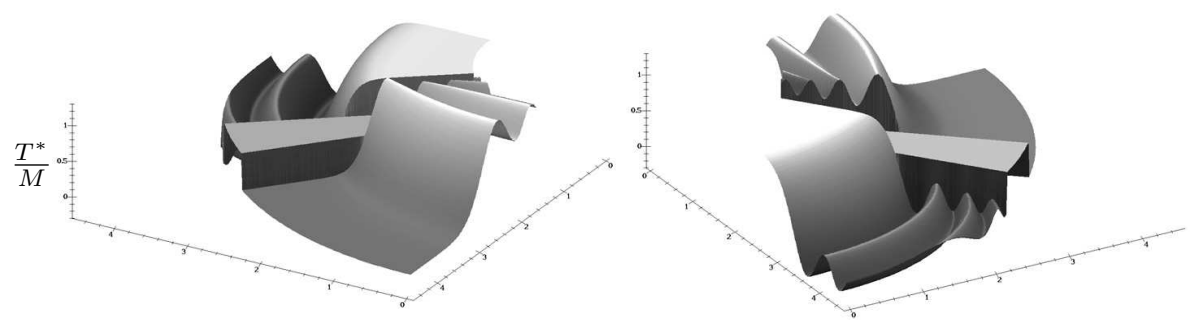

Figure 3. The dependence of the quasi-front type on the angle $\theta$ of wave propagation. Table 1 summarizes utilized material parameters.

may change as the propagation angle varies. This phenomenon is further illuminated by the diagrams presented in figure 3 , which show the traction against space coordinate $\xi_{n}$ at $\tau=3$. A polar-like system of coordinates was used with the angle equal to the angle of wave propagation $\theta$ and the distance from pole equal to $\xi_{n}$. We remark, that since our asymptotic 
model is not valid when $\mathcal{F}^{(2)}$ is small, the corresponding values of traction were replaced on the diagrams with 0.5 to clearly distinguish these areas.

It is important to note that these diagrams are not to be considered as the possible traction distributions generated by a point load, although the solution for point load problem will feature similar change of the wave type. We also note that the behaviour at the transition from one type of wave front to another can not be properly predicted by this model as it corresponds to vanishing of $\mathcal{F}^{(2)}$ and, consequently, the third order derivative term in the governing equation (5.11). The corresponding values of traction were replaced on the diagrams with 0.5 to clearly distinguish these transition regions. An analysis of within the vicinity of the transition region may only be carried out by resorting to the higher order approximation and calculation of a third order governing equation.

The work of A. V. Pichugin is supported by the U. K. Overseas Research Student Award and by the award from the University of Salford. These awards are gratefully acknowledged.

\section{References}

Cole, J. D. 1968 Perturbation methods in applied mathematics. Blaisdell, Waltham, MA.

Dowaikh, M. A. \& Ogden, R. W. 1990 On surface waves and deformations in a pre-stressed incompressible elastic solid. IMA Jl. Appl. Math. 44, 261-284.

Green, A. E. 1962 On the linear theory of thin elastic shells. Proc. R. Soc. Lond. A266, $143-160$

Kaplunov, J. D., Kossovich, L. Y. \& Nolde, E. V. 1998 Dynamics of thin walled elastic bodies. New York: Academic Press.

Kaplunov, J. D., Nolde, E. V. \& Rogerson, G. A. 2000 A low frequency model for dynamic motion in pre-stressed incompressible elastic structures. Proc. R. Soc. Lond. A456, 25892610 .

Kaplunov, J. D., Nolde, E. V. \& Rogerson, G. A. 2001 An asymptotically consistent model for long wave high frequency motion in a pre-stressed elastic plate. Mech. Math. Solids To appear.

Kevorkian, J. 1989 Partial differential equations: analytical solution techniques. Pacific Grove, California: Wadsworth \& Brooks/Cole Advanced Books \& Software. 
Ogden, R. W. 1984 Non-linear elastic deformations. New York: Ellis Horwood.

Ogden, R. W. \& Sotiropoulos, D. A. 1995 On interfacial waves in pre-stressed layered incompressible solids. Proc. R. Soc. Lond. A450, 319-341.

Pichugin, A. V. \& Rogerson, G. A. 2001a Dynamic model of a pre-stressed incompressible elastic layer in the vicinity of shear resonance. Submitted for publication.

Pichugin, A. V. \& Rogerson, G. A. 2001b A two-dimensional model for extensional motion of a pre-stressed incompressible elastic layer near cut-off frequencies. IMA Jl. Appl. Math. 66, 357-385.

Rogerson, G. A. \& Fu, Y. B. 1995 An asymptotic analysis of the dispersion relation of a pre-stressed incompressible elastic plate. Acta Mechanica 111, 59-77.

Rogerson, G. A. \& Sandiford, K. J. 1997 Flexural waves in pre-stressed incompressible elastic composites. Quart. Jl. Mech. Appl. Math. 50(4), 597-624.

Rogerson, G. A. \& Sandiford, K. J. 1999 Harmonic wave propagation along a non-principal direction in a pre-stressed elastic plate. Int. Jl. Eng. Sci. 37, 1663-1691.

Spencer, A. J. M. 1980 Continuum mechanics. Longman Scientific \& Technical. 